\title{
Use of population input functions for reduced scan duration whole-body Patlak ${ }^{18}$ F-FDG PET imaging
}

\author{
Joyce van Sluis ${ }^{1 *} \mathbb{D}$, Maqsood Yaqub ${ }^{2}$, Adrienne H. Brouwers' ${ }^{1}$, Rudi A. J. O. Dierckx', Walter Noordzij ${ }^{1}$ and
} Ronald Boellaard ${ }^{1,2}$

\footnotetext{
*Correspondence: j.van.sluis@umcg. $\mathrm{nl}$

${ }^{1}$ Department of Nuclear Medicine and Molecular Imaging, University Medical Center Groningen, University of Groningen, Hanzeplein 1, 9713 GZ Groningen, The Netherlands

Full list of author information is available at the end of the article
}

Abstract: Whole-body Patlak images can be obtained from an acquisition of first 6 min of dynamic imaging over the heart to obtain the arterial input function (IF), followed by multiple whole-body sweeps up to $60 \mathrm{~min}$ pi. The use of a populationaveraged IF (PIF) could exclude the first dynamic scan and minimize whole-body sweeps to 30-60 min pi. Here, the effects of (incorrect) PIFs on the accuracy of the proposed Patlak method were assessed. In addition, the extent of mitigating these biases through rescaling of the PIF to image-derived IF values at 30-60 min pi was evaluated.

Methods: Using a representative IF and rate constants from the literature, various tumour time-activity curves (TACS) were simulated. Variations included multiplication of the IF with a positive and negative gradual linear bias over 60 min of 5, 10, 15, 20, and 25\% (generating TACs using an IF different from the PIF); use of rate constants $\left(K_{1}, K_{3}\right.$, and both $K_{1}$ and $\left.k_{2}\right)$ multiplied by $2,1.5$, and 0.75 ; and addition of noise $(\mu=$ 0 and $\sigma=5,10$ and 15\%). Subsequent Patlak analysis using the original IF (representing the PIF) was used to obtain the influx constant $\left(K_{i}\right)$ for the differently simulated TACs. Next, the PIF was scaled towards the (simulated) IF value using the 30-60-min pi time interval, simulating scaling of the PIF to image-derived values. Influence of variabilities in IF and rate constants, and rescaling the PIF on bias in $K_{i}$ was evaluated.

Results: Percentage bias in $K_{i}$ observed using simulated modified IFs varied from 16 to $16 \%$ depending on the simulated amplitude and direction of the IF modifications. Subsequent scaling of the PIF reduced these $K_{i}$ biases in most cases (287 out of 290) to $<5 \%$.

Conclusions: Simulations suggest that scaling of a (possibly incorrect) PIF to IF values seen in whole-body dynamic imaging from 30 to 60 min pi can provide accurate Ki estimates. Consequently, dynamic Patlak imaging protocols may be performed for 30-60 min pi making whole-body Patlak imaging clinically feasible.

Keywords: Patlak, PET/CT, Dynamic imaging, Population input function, Scan time 


\section{Introduction}

Positron emission tomography integrated with computed tomography (PET/CT) imaging using ${ }^{18} \mathrm{~F}$-2-fluoro-2-deoxy-D-glucose $\left({ }^{18} \mathrm{~F}\right.$-FDG) is widely used in oncology for diagnosis, staging, and treatment response evaluation [1-11]. The standardized uptake value (SUV), a semi-quantitative metric derived from PET images, is most commonly used as a surrogate of metabolic activity for quantifying ${ }^{18}$ F-FDG tumour uptake [1]. SUV can be derived from static PET acquisitions, typically initiated $1 \mathrm{~h}$ post-injection (pi) where every bed position is scanned once for $2-5 \mathrm{~min}[1,12]$. Through standardization methods regarding patient preparation (to avoid, e.g., high plasma glucose), PET acquisition settings, image reconstruction, and analysis methods, SUV variability can be limited to a great extent $[1,13,14]$. However, quantitative accuracy of SUV can also be influenced by changes in plasma kinetics due to treatment possibly causing inaccurate assessments [15-17].

Dynamic PET imaging allows for spatiotemporal activity concentration distribution measurement which can provide voxel-wise metabolic information when used by tracer pharmacokinetic modelling methods, for example, full kinetic analysis, i.e., Patlak analysis [18-21].

Up to recently, dynamic PET imaging was mainly performed using single-bed/singleaxial field-of-view acquisitions. Currently, with state-of-the-art PET/CT systems, whole-body dynamic (Patlak) images can be obtained from a combined acquisition of first 6 min of dynamic imaging over the heart to obtain the arterial input function (IF) followed by multiple whole-body sweeps up to $60 \mathrm{~min}$ pi. This procedure followed by a standard static whole-body PET, however, can take a total examination time to $75 \mathrm{~min}$ [22], including the time needed for patient positioning and CT procedure. Initial PET examinations using whole-body Patlak imaging showed a high frequency of patients' inability to comply with the long scan duration required for the protocol.

The use of a population-averaged input function (PIFs) could obviate the need for the first dynamic scan and minimize whole-body sweeps to an interval of 30-60 min pi [23] making whole-body dynamic Patlak imaging clinically feasible. There have been various studies in which using a PIF is explored in oncological whole-body dynamic ${ }^{18}$ F-FDG imaging [21, 23-26]. Promising results were obtained in comparison with using an arterial IF and an image derived IF; however, further evaluation of microparameter estimation (such as $k_{3}$ and (if it exists) $k_{4}$ ) is recommended before implementation [24]. Therefore, given that this approach may introduce some inaccuracy, this study explored the effects of (incorrect) PIFs on the accuracy of the proposed Patlak method using various simulations including variations in rate constants. In addition, the extent of mitigating these biases through rescaling of the PIF to image-derived values at $30-60 \mathrm{~min}$ pi was evaluated.

\section{Materials and methods}

To explore the effects of (possibly incorrect) PIFs on the accuracy of Patlak analysis based on dynamic whole-body PET acquisition from 30 to $60 \mathrm{~min}$ pi, various tumour time-activity curves (TACs) were simulated. To this aim, a representative IF from previously acquired data (acquisition and processing described in [21]) was used as the PIF as well as rate constants based on literature: $K_{1}$ was $0.301 \mathrm{~min}^{-1}, k_{2}$ was $0.600 \mathrm{~min}^{-1}$, and $k_{3}$ was $0.047 \mathrm{~min}^{-1}[18,27]$. TACs were created according to Eq. 1: 


$$
C_{\text {tissue }}=C_{\text {blood }} \otimes\left[k_{2} e^{-\left(k_{2}+k_{3}\right) t}+k_{3}\right] \frac{K_{1}}{k_{2}+k_{3}}
$$

Here, $C_{\text {blood }}$ represents the original representative left ventricle arterial IF, and $C_{\text {tissue }}$ is the obtained TAC. The PET TAC was then generated using Eq. 2:

$$
C_{\mathrm{PET}}=\left(1-V_{b}\right) \times C_{\text {tissue }}+V_{b} \times C_{\text {blood }}
$$

with $V_{b}$ equal to the blood volume fraction.

Simulations were performed in the Python programming language using an in-house written code.

Through the multiplication of the IF with a positive and negative gradual linear bias using different slopes over $60 \mathrm{~min}$, variations in IF were simulated. Differences in slope steepness included a positive and negative gradual linear bias from one to $5,10,15,20$, and $25 \%$ multiplied by the IF, e.g., the IF was multiplied by a positive and negative linear function starting at $y=1$ to $y=1.25$ and starting at $y=1$ to $y=0.75$ in the case of $25 \%$ bias. These simulations were also reversed, e.g., the IF was multiplied by a positive and negative linear function starting at $y=1.25$ to $y=1$ and starting at $y=0.75$ to $y=$ 1 in the case of $25 \%$ bias. Variations in IF accounted for a total number of 35 simulations.

Other variations that were combined with the gradual bias from one to different slope sizes included the use of rate constants $\left(K_{1}, k_{3}\right.$, and both $K_{1}$ and $\left.k_{2}\right)$ multiplied by $2,1.5$, and 0.75 (fitting the mean and exceeding the range of rate constants observed in clinical data [27]). These rate constant variations combined with IF modifications accounted for a total number of 180 simulations. In addition, noise was added to the IF modifications ( $\mu=0$ and $\sigma=5,10$, and 15\% simulating high, medium, and low noise equivalent count rate [28], respectively) to account for the differences between PET/CT systems and reconstruction settings. The noise additions combined with IF modifications accounted for a total number of 75 simulations. For the simulations described above, the blood volume fraction was fixed at $8.9 \%$ [27].

To show the extent to which incorrect PIFs influence the accuracy of Patlak analysis, analyses using the original IF (representing the PIF) were used to obtain the influx constant $\left(K_{i}\right)$ for the differently simulated TACs. Subsequently, the incorrect PIFs were scaled towards the correct value using the 30-60-min pi time-interval simulating scaling of the PIF to image-derived values. After rescaling, $K_{i}$ were obtained using these adjusted PIFs to see the extent to which previously obtained biases in $K_{i}$ could be mitigated. A schematic overview regarding the modification and rescaling of the IF is shown in Fig. 1.

\section{Results}

In total, 290 variations were simulated (35 gradual in or decrease of the IF and reversed, 180 rate constant variations, and 75 noise simulations). Percentage bias in $K_{i}$ using simulated incorrect IFs varied from -16 to $16 \%$ depending on the simulation type, amplitude, addition of noise, and direction of the IF modifications. Subsequent scaling of the PIF reduced these $K_{i}$ biases in most cases to between -3 and $4 \%$ for the gradual in and decrease of the IF and reversed, between -3.7 and $4 \%$ for the rate 


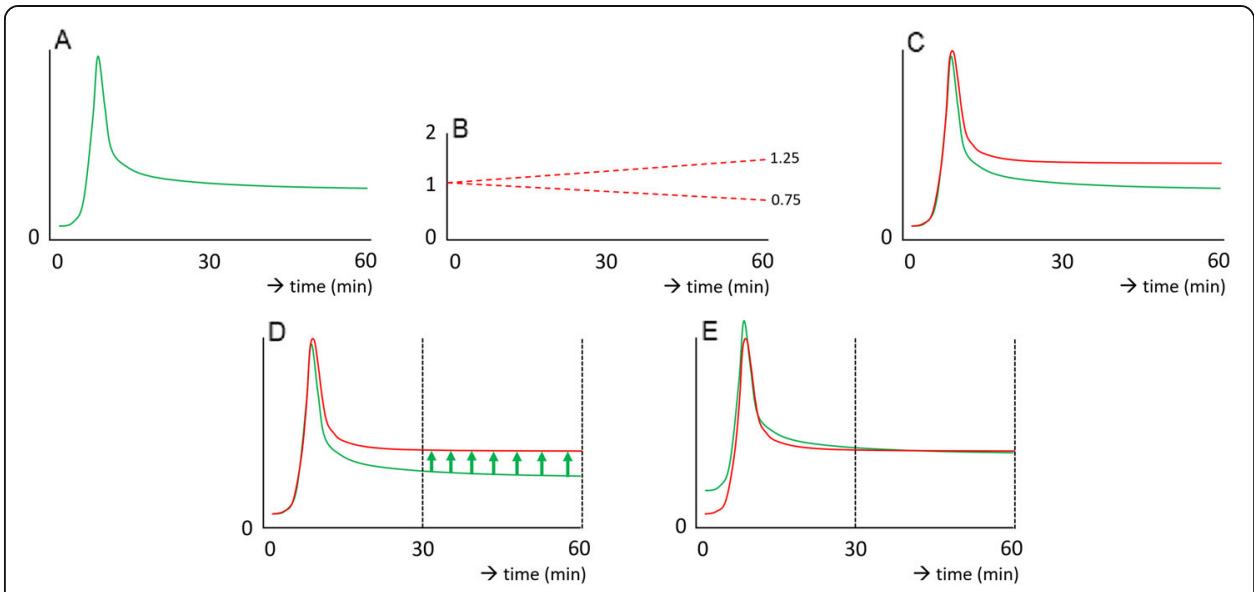

Fig. 1 Schematic overview of PIF (green) with a gradual linear modification (dashed red) towards a variety of (incorrect) IFs (red) (a-c) and subsequent rescaling of the PIF at 30-60 min pi (d, e)

constant variations combined with gradual in and decrease of the IF, and between -2.4 and $3 \%$ for the noise addition combined with gradual in and decrease of the IF.

Figure 2 shows the influence of positive, negative, and reversed gradual input function modification over $60 \mathrm{~min}$ of $5,10,15,20$, and $25 \%$ on the percentage difference in $K_{i}$ before and after rescaling of the PIF. The effect of variations in rate constants combined with a gradual modification of the IF on the percentage difference in $K_{i}$ before and after rescaling of the PIF is shown in Fig. 3. For the influence of added noise combined with a gradual modification of the IF on the percentage difference in $K_{i}$ before and after rescaling of the PIF, see Fig. 4.

\section{Discussion}

Tumour quantification using SUVpeak following the PERCIST guidelines describes stable metabolic disease as an increase or decrease in SUVpeak of less than 30\% [29]. However, SUV can be affected by variations (between subjects or longitudinally) of the plasma kinetics which may cause inaccuracies in clinical assessments. Nevertheless, Patlak analysis also remains an estimate of the tumour's kinetic behaviour and may be biassed in case of large blood volume fractions for example in cases with bulky tumours [21]; this should be taken into account. Based on the reasoning as described above, we determined that an accuracy level of $5 \%$ is acceptable, i.e., well within repeatability levels (which are in the order of 10 to $15 \%$ [30-32]).

In most cases, rescaling of the PIF reduced $K_{i}$ biases to $<5 \%$. However, there were three incidences (out of 290) which resulted in remaining biases $>5 \%$ after rescaling: the combination of $25 \%$ gradual IF modification and $k_{3} * 2$ resulted in a remaining $K_{i}$ bias of $8 \%$, the combination of $25 \%$ gradual IF modification and $k_{3}{ }^{*} 1.5$ resulted in a remaining $K_{i}$ bias of $7 \%$, and the combination of $20 \%$ gradual IF modification and $k_{3}{ }^{* 2}$ resulted in a remaining bias in $K_{i}$ of $6 \%$. When using PIFs for whole-body Patlak imaging in patients with high nuclear grade and/or high proliferation activity tumours (associated with higher $k_{3}$ [33]), this should be taken into account.

Please note that when using Patlak analysis, bias may occur even with a perfectly correct IF. Bias in the Patlak analysis may occur as a result of not incorporating the fractional blood volume in the Patlak equations. Blood volume fractions of 0, 8.9, and 20\% 


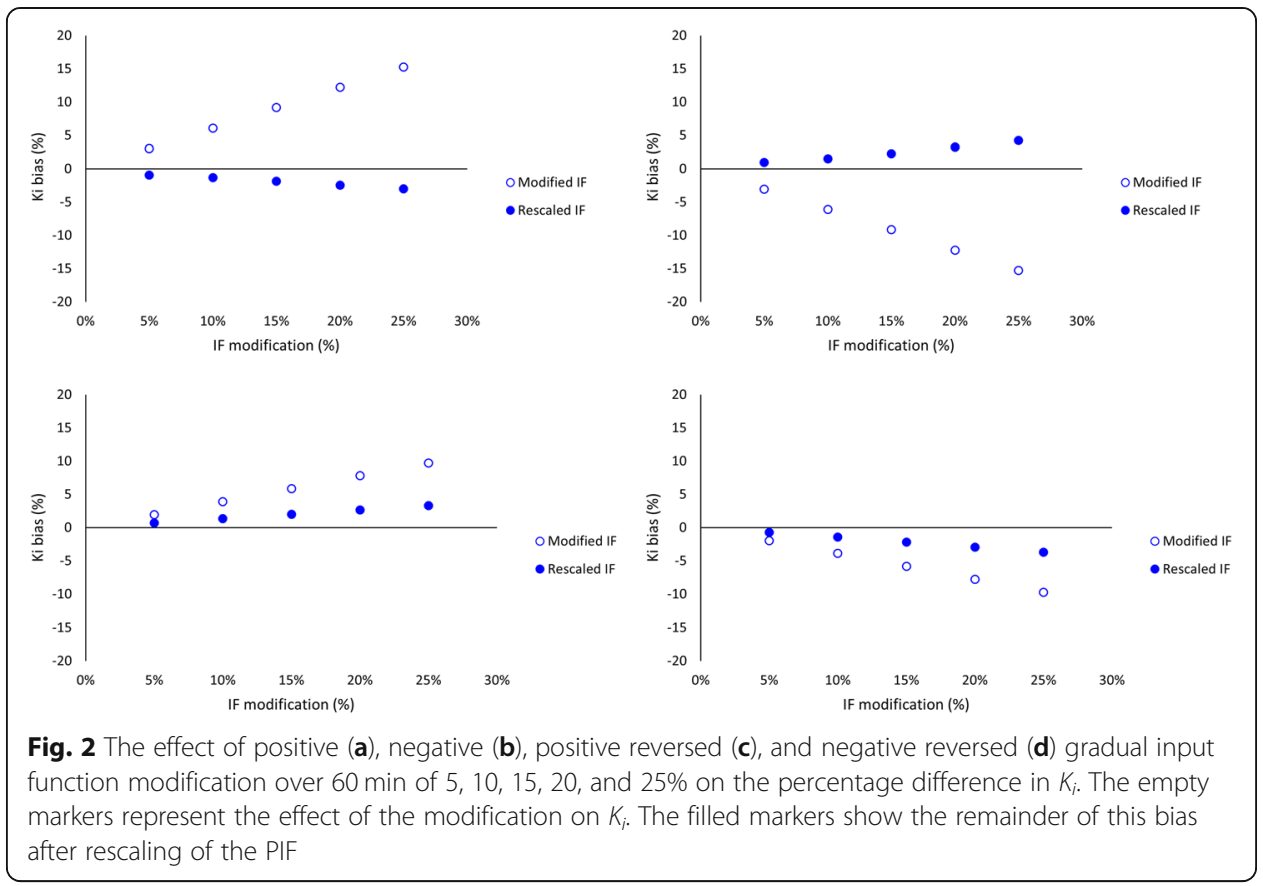

resulted in a Patlak $K_{i}$ bias of $-1.2,-10$, and $-20 \%$, respectively (data not shown). This indicates that the $K_{i}$ bias through the use of a (possibly incorrect, but rescaled) PIF is small compared to possible biases in case of large blood volume fractions. With regard to the acceptable accuracy level of $5 \%$ when using a PIF for estimating the tumour kinetic behaviour, the acceptable total bias would be $25 \%$, which is just within the PERC IST recommendations [29]. Yet, for most tumours, the blood volume fraction is typically smaller than $10 \%$ [27] resulting in a total acceptable bias of $15 \%$, so again, well within the limits of agreement regarding repeatability and PERCIST criteria.

A similar study to develop a simplified Patlak protocol through using PIFs based on clinical data was performed by S. Yao et al. [26]. Here, similar biases (from -20 to $20 \%$ ) were induced to a representative IF to simulate and explore the effect of possible errors in PIFs when applied at $20 \mathrm{~min}$ pi. They concluded that whenever the IF modification remains below 20\%, quantitative inaccuracy regarding $K_{i}$ would be around $4 \%$ [26], which is in line with the results of our simulations.

Another study that explored the alternative of using a PIF instead of arterial blood sampling found a very high correlation between the two methods [23]. The addition of variability in rate constants and noise in our study provides a more comprehensive and realistic reflection of the possible range in kinetic parameters seen in tumours.

\section{Conclusion}

Simulations suggest that scaling of a possibly incorrect PIF to (image derived) IF values seen in whole-body dynamic imaging from 30 to $60 \mathrm{~min}$ pi could be a good strategy to obtain accurate $K_{i}$ estimates. Consequently, dynamic Patlak imaging protocols may be performed for 30-60 min pi making whole-body Patlak imaging clinically feasible. 


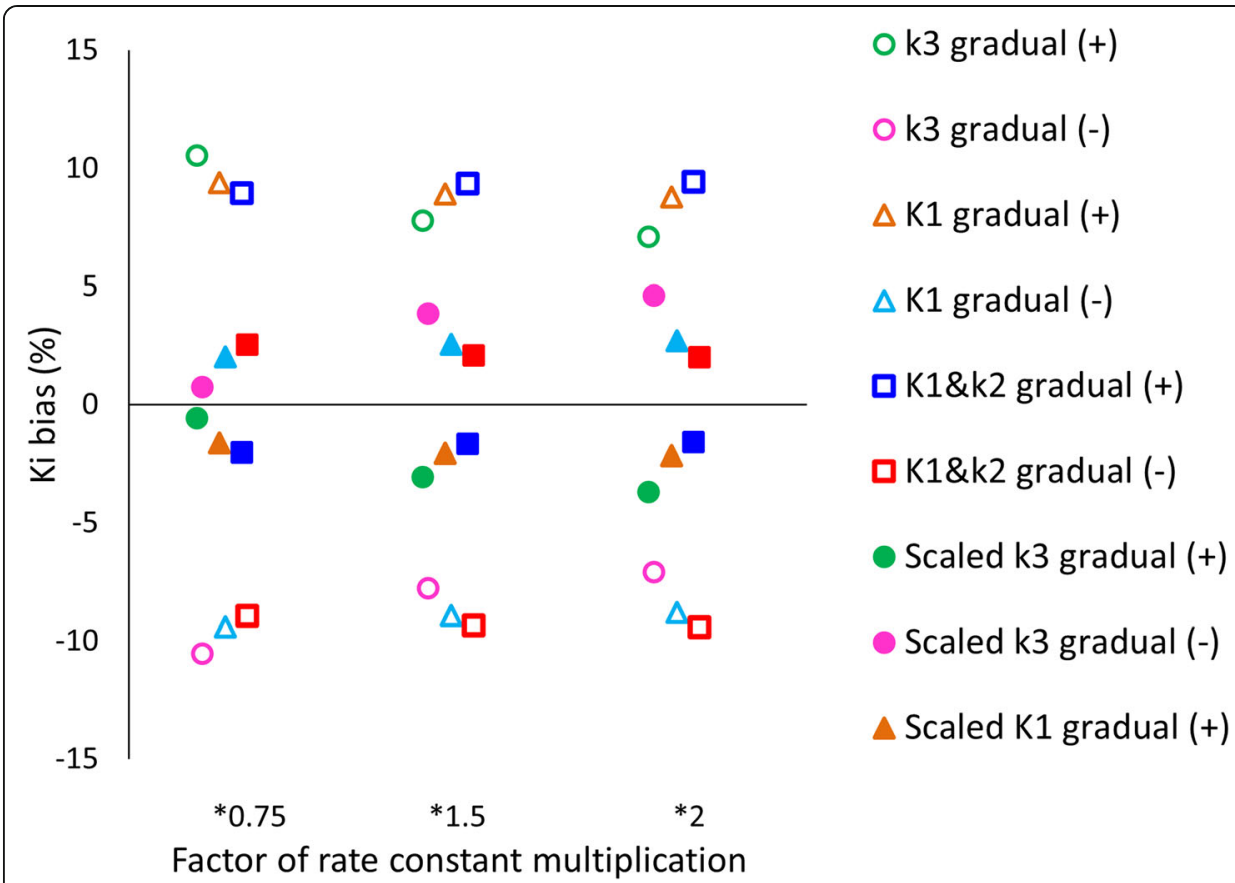

Fig. 3 The effect of variations in rate constants combined with 15\% gradual modification of the IF on the percentage difference in $K_{i}$. The empty markers represent the effect of the modification on $K_{i}$. The filled markers show the remainder of this bias after rescaling of the PIF

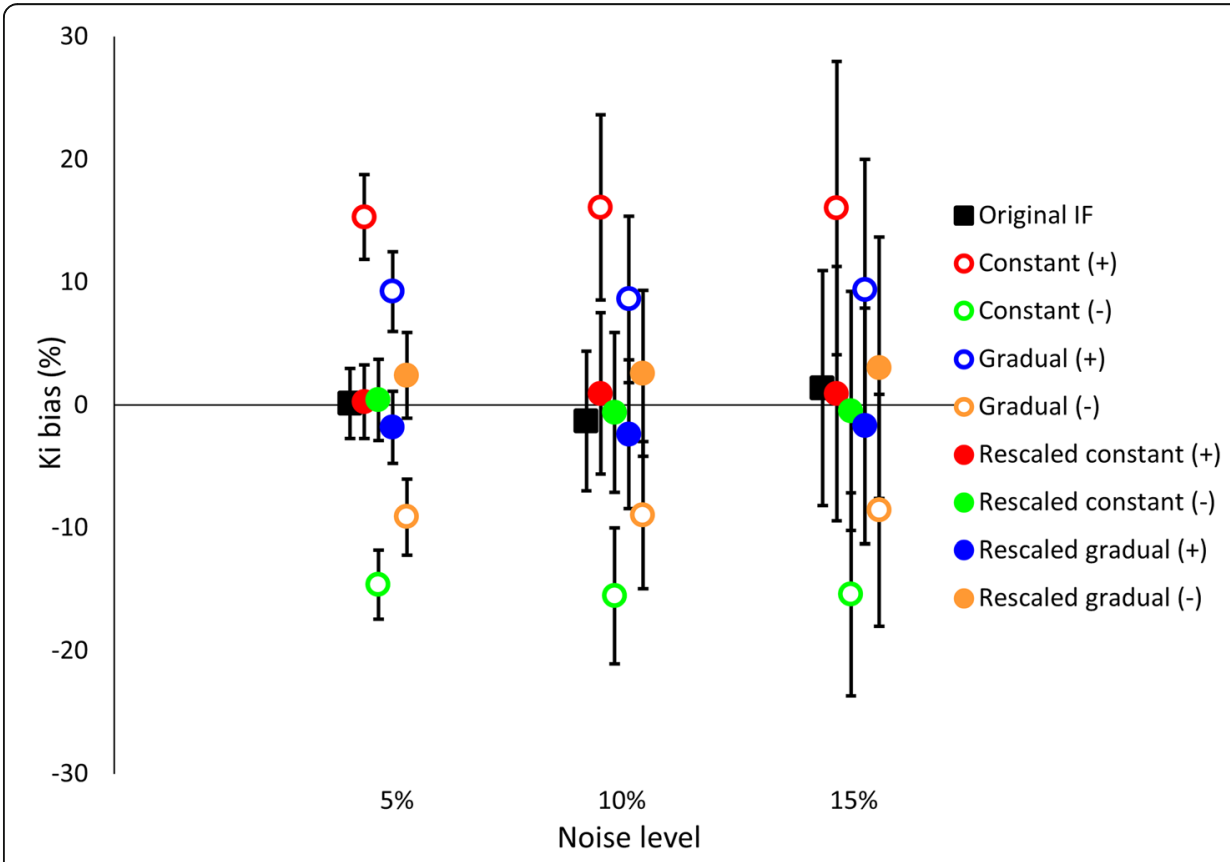

Fig. 4 The effect of added noise combined with 15\% gradual modification of the IF on the percentage difference in $K_{i}$. The original input function with added noise is represented by the filled black marker. The empty markers represent the effect of the modification on $K_{i}$. The filled markers show the remainder of this bias after rescaling of the PIF 
Acknowledgements

Not applicable.

\section{Authors' contributions}

$J S, M Y$, and RB contributed to the study concepts and design. JS, MY, and RB contributed to the data generation, analysis, and interpretation. JS, MY, and RB contributed to the manuscript preparation. JS, MY, AB, RD, WN, and RB contributed to the manuscript review and editing. All authors read and approved the final manuscript.

\section{Funding}

Not applicable.

\section{Availability of data and materials}

All data generated during the current study are available from the corresponding author on reasonable request.

Ethics approval and consent to participate

Not applicable.

\section{Consent for publication}

Not applicable.

\section{Competing interests}

The authors declare that they have no competing interests.

\section{Author details}

'Department of Nuclear Medicine and Molecular Imaging, University Medical Center Groningen, University of Groningen, Hanzeplein 1, 9713 GZ Groningen, The Netherlands. 'Department of Radiology and Nuclear Medicine, Cancer Center Amsterdam, Amsterdam UMC, Vrije Universiteit Amsterdam, Amsterdam, The Netherlands.

Received: 16 September 2020 Accepted: 22 January 2021

Published online: 05 February 2021

\section{References}

1. Boellaard R, Delgado-Bolton R, Oyen WJG, Giammarile F, Tatsch K, Eschner W, et al. FDG PET/CT: EANM procedure guidelines for tumour imaging: version 2.0. Eur J Nucl Med Mol Imaging. 2014;42:328-54.

2. Avril NE, Weber WA. Monitoring response to treatment in patients utilizing PET. Radiol Clin N Am. 2005:43:189-204.

3. Gupta T, Master Z, Kannan S, Agarwal JP, Ghsoh-Laskar S, Rangarajan V, et al. Diagnostic performance of post-treatment FDG PET or FDG PET/CT imaging in head and neck cancer: a systematic review and meta-analysis. Eur J Nucl Med Mol Imaging. 2011;38:2083-95.

4. Slomka PJ, Pan T, Germano G. Recent advances and future progress in PET instrumentation. Semin Nucl Med. Elsevier. 2016;46:5-19.

5. Lv YL, Yuan DM, Wang K, Miao XH, Qian Q, Wei SZ, et al. Diagnostic performance of integrated positron emission tomography/computed tomography for mediastinal lymph node staging in non-small cell lung cancer: a bivariate systematic review and meta-analysis. J Thorac Oncol. International Association for the Study of Lung Cancer. 2011;6: $1350-8$

6. Hsu DFC, llan E, Peterson WT, Uribe J, Lubberink M, Levin CS. Studies of a next-generation silicon-photomultiplier-based time-of-flight PET/CT system. J Nucl Med. 2017:58:1511-8.

7. Townsend DW. Dual-modality imaging: combining anatomy and function. J Nucl Med. 2008;49:938-55.

8. Boellaard R, Oyen WJG, Hoekstra CJ, Hoekstra OS, Visser EP, Willemsen AT, et al. The Netherlands protocol for standardisation and quantification of FDG whole body PET studies in multi-centre trials. Eur J Nucl Med Mol Imaging. 2008:35:2320-33.

9. Bastiaannet E, Groen B, Jager PL, Cobben DCP, van der Graaf WTA, Vaalburg W, et al. The value of FDG-PET in the detection, grading and response to therapy of soft tissue and bone sarcomas; a systematic review and meta-analysis. Cancer Treat Rev. 2004:30:83-101.

10. Fletcher JW, Djulbegovic B, Soares HP, Siegel BA, Lowe VJ, Lyman GH, et al. Recommendations on the use of 18F-FDG PET in oncology. J Nucl Med. 2008:49:480-508.

11. De Geus-Oei LF, Van Der Heijden HFM, Corstens FHM, Oyen WJG. Predictive and prognostic value of FDG-PET in nonsmall-cell lung cancer. A systematic review. Cancer. 2007;110:1654-64.

12. Castell F, Cook GJR. Quantitative techniques in 18FDG PET scanning in oncology. Br J Cancer. 2008;98:1597-601.

13. Weber WA. Use of PET for monitoring cancer therapy and for predicting outcome. J Nucl Med. 2005:46:983-95.

14. Shankar LK, Hoffman JM, Bacharach S, Graham MM, Karp J, Lammertsma AA, et al. Consensus recommendations for the use of 18F-FDG PET as an indicator of therapeutic response in patients in National Cancer Institute Trials. J Nucl Med. 2006:47:1059-66.

15. Freedman NMT, Sundaram SK, Kurdziel K, Carrasquillo JA, Whatley M, Carson JM, et al. Comparison of SUV and Patlak slope for monitoring of cancer therapy using serial PET scans. Eur J Nucl Med Mol Imaging. 2003;30:46-53.

16. Huang SC. Anatomy of SUV. Nucl Med Biol. 2000;27:643-6.

17. Lammertsma AA, Hoekstra CJ, Giaccone G, Hoekstra OS. How should we analyse FDG PET studies for monitoring tumour response? Eur J Nucl Med Mol Imaging. 2006;33(Supplement 13):16-21.

18. Karakatsanis NA, Lodge MA, Tahari AK, Zhou Y, Wahl RL, Rahmim A. Dynamic whole body PET parametric imaging: I. Concept, acquisition protocol optimization and clinical application Nicolas. Phys Med Biol. 2014;49:1012-6.

19. Patlak CS, Blasberg RG. Graphical evaluation of blood-to-brain transfer constants from multiple-time uptake data. Generalizations. J Cereb Blood Flow Metab. 1985;5:584-90. 
20. Karakatsanis NA, Zhou Y, Lodge MA, Casey ME, Wahl RL, Zaidi H, et al. Generalized whole-body Patlak parametric imaging for enhanced quantification in clinical PET. Phys Med Biol. 2015;60:8643-73.

21. Cheebsumon P, Velasquez LM, Hoekstra CJ, Hayes W, Kloet RW, Hoetjes NJ, et al. Measuring response to therapy using FDG PET: semi-quantitative and full kinetic analysis. Eur J Nucl Med Mol Imaging. 2011;38:832-42.

22. Zaidi H, Karakatsanis N. Nuclear medicine: physics special feature review article: towards enhanced pet quantification in clinical oncology. Br J Radiol. 2018;91:20170508.

23. Naganawa M, Gallezot J-D, Shah V, Mulnix T, Chen M-K, Smith A, et al. Assessment of population-based input functions for the Patlak plot using whole body 18F-FDG PET imaging. EJNMMI Phys. 2020;7:67 Published 2020 Nov 23.

24. Vriens D, De Geus-Oei LF, Oyen WJG, Visser EP. A curve-fitting approach to estimate the arterial plasma input function for the assessment of glucose metabolic rate and response to treatment. J Nucl Med. 2009;50:1933-9.

25. Hunter GJ, Hamberg LM, Alpert NM, Choi NC, Fischman AJ. Simplified measurement of deoxyglucose utilization rate. J Nucl Med. 1996;37:950-5.

26. Yao S, Feng T, Zhao Y, Wu R, Wang R, Wu S, et al. Simplified protocol for whole body Patlak parametric imaging with 18 F-FDG PET/CT: feasibility and error analysis. Med Phys. 2020. https://doi.org/10.1002/mp.14187 Published 2020 Apr 17.

27. Vriens D, Disselhorst JA, Oyen WJG, De Geus-Oei LF, Visser EP. Quantitative assessment of heterogeneity in tumor metabolism using FDG-PET. Int J Radiat Oncol Biol Phys. 2012;82:725-31.

28. Boellaard R, Krak NC, Hoekstra OS, Lammertsma AA. Effects of noise, image resolution, and ROI definition on the accuracy of standard uptake values: a simulation study. J Nucl Med. 2004;45:1519-27.

29. Joo Hyun O, Lodge MA, Wahl RL. Practical PERCIST: a simplified guide to PET response criteria in solid tumors 1.0. Radiology. 2016;280:576-84

30. Weber WA, Gatsonis CA, Mozley PD, Hanna LG, Shields AF, Aberle DR, et al. Repeatability of 18F-FDG PET/CT in advanced non-small cell lung cancer: prospective assessment in 2 multicenter trials. J Nucl Med. 2016:56:1137-43.

31. Kramer GM, Frings V, Hoetjes N, Hoekstra OS, Smit EF, De Langen AJ, et al. Repeatability of quantitative whole-body 18F-FDG PET/CT uptake measures as function of uptake interval and lesion selection in non-small cell lung cancer patients. J Nucl Med. 2016;57:1343-9.

32. De Langen AJ, Vincent A, Velasquez LM, Van Tinteren H, Boellaard R, Shankar LK, et al. Repeatability of 18F-FDG uptake measurements in tumors: a metaanalysis. J Nucl Med. 2012;53:701-8.

33. Kajáry K, Lengyel Z, Tőkés AM, Kulka J, Dank M, Tőkés T. Dynamic FDG-PET/CT in the initial staging of primary breast cancer: clinicopathological correlations. Pathol Oncol Res. 2020;26:997-1006.

\section{Publisher's Note}

Springer Nature remains neutral with regard to jurisdictional claims in published maps and institutional affiliations.

\section{Submit your manuscript to a SpringerOpen ${ }^{\circ}$ journal and benefit from:}

- Convenient online submission

- Rigorous peer review

Open access: articles freely available online

- High visibility within the field

Retaining the copyright to your article

Submit your next manuscript at $\boldsymbol{\sim}$ springeropen.com 